\title{
Kotherapeuten als Verhaltenstherapeuten?
}

\author{
Ulrich Voderholzer \\ Abteilung für Psychosomatik, Schön Klinik Roseneck, Prien, Deutschland
}

In psychiatrischen und verhaltenstherapeutischen Kliniken werden unter «Kotherapeuten» unter anderem Krankenschwestern und -pfleger, Sport- und Ergotherapeuten, sowie Musik- und Kunsttherapeuten subsumiert. Darunter versteht man das fachliche Personal, das die Ärzte, Psychologen und Psychotherapeuten in der Therapie der Patienten unterstützt und eigene Schwerpunkte in den Gesamtbehandlungsplan einbringt. Während z.B. Ergotherapeuten mit ihrem Ansatz vor allem auf die Verbesserung und den Wiederaufbau des «gesunden Lebens» abzielen und damit einen wichtigen Beitrag zur Herstellung der Leistungsfähigkeit und Partizipation im Alltag leisten [Linden und Gehrke, 2013], werden Krankenschwestern und -pfleger, die in ihrer Ausbildung meist eher die Behandlung und Versorgung körperlicher Erkrankungen von Patienten erlernt haben, häufig in aktuellen psychischen Krisensituationen der Patienten mit therapeutischen Aufgaben konfrontiert.

\section{Sind Kotherapeuten in der Lage, verhaltenstherapeutisch zu arbeiten?}

Gerade in den USA stellen Kotherapeuten eine wichtige Unterstützung im medizinischen Versorgungssystem dar und es gibt bereits eine Vielzahl an verschiedenen Aus- und Weiterbildungsprogrammen. Neben den vielfältigen Einsatzmöglichkeiten der Kotherapeuten stellen vor allem die geringeren Kosten, die kürzeren Ausbildungszeiten und die große Anzahl an Krankenschwestern und -pflegern Gründe für die steigenden Weiterbildungsangebote dar [Marks et al., 1975]. Gourney und Kollegen [2000] sehen in den letzten Jahren vor allem in England positive Entwicklungen durch das steigende Angebot an Supervision, auch wenn es nach dieser Studie noch immer zu wenige Kotherapeuten mit einer Zusatzausbildung gibt. Die Schwerpunkte der kotherapeutisch tätigen Krankenschwestern und -pfleger liegen nach dieser Studie in der stationären Versorgung mit einem verhaltenstherapeuti- schen Schwerpunkt. Auch frühere Studien [Newell und Gourney, 1994] zeigten bereits, dass fachlich qualifizierte Kotherapeuten vor allem kurze und verhaltenstherapeutische Interventionen übernehmen können. In ihrem Review unterstützten Zauszniewski und Kollegen [2007] diese Ergebnisse, indem sie nachwiesen, dass die meisten kontrollierten Studien mit Kotherapeuten im Bereich der verhaltenstherapeutischen Interventionen durchgeführt wurden. Dabei konnten positive Effekte beim Einsatz von Kotherapeuten vor allem im Bereich Psychoedukation, bei kognitiven Interventionen und Verhaltensmodifikationen festgestellt werden.

\section{Gibt es spezielle Aus- und Weiterbildungscurricula für Kotherapeuten?}

In Europa gibt es derzeit noch keine einheitliche Regelung über die Ausbildung und den Einsatz von Kotherapeuten in der psychotherapeutischen Versorgung [Ward, 2012]. In Deutschland werden Kotherapeuten an vielen psychosomatischen und psychiatrischen Kliniken eingesetzt; spezielle Ausbildungsprojekte gibt es jedoch auch hier noch wenige. Viele Weiterbildungen erfolgen in Deutschland klinikintern in Kooperation mit psychotherapeutischen Ausbildungsinstituten, sodass die Qualität der Ausbildungen zwar uneinheitlich, dafür aber häufig sehr gut ist.

Auch in der Psychosomatischen Schön Klinik Roseneck in Prien am Chiemsee erfolgt die medizinische und psychologische Versorgung der Patienten nicht nur durch Ärzte und Psychologen/Psychotherapeuten, sondern auch durch Krankenschwestern und -pfleger als Kotherapeuten sowie Sport- und Gestaltungstherapeuten. Gerade die Krankenschwestern und -pfleger erleben die Patienten während ihres Klinikaufenthalts dabei sehr intensiv und sind die ersten Ansprechpartner, wenn es Anliegen oder auch Probleme gibt. Dabei werden sie immer wieder mit krisenhaften Zuständen der Patienten konfrontiert und sollen die Patienten unterstützen, indem sie mit

\section{KARGER \\ Fax +497614520714 \\ Information@Karger.com}

www.karger.com (c) 2013 S. Karger GmbH, Freiburg

$1016-6262 / 13 / 0231-0004 \$ 38.00 / 0$

Accessible online at:

www.karger.com/ver
Prof. Dr. med. Ulrich Voderholzer

Schön Klinik Roseneck

Am Roseneck 6, 83209 Prien am Chiemsee, Deutschland

UVoderholzer@schoen-kliniken.de 
ihnen Tagebücher ausfüllen oder sie bei Expositionen anleiten. Zur Schulung und Verbesserung der psychotherapeutischen Kenntnisse nehmen die Kotherapeuten an einem speziellen wöchentlichen Curriculum mit $186 \mathrm{~h}$ teil, das von Ärzten und psychologischen Psychotherapeuten mit verhaltenstherapeutischer Ausbildung geleitet wird und in dem das Basiswissen der verhaltenstherapeutischen Arbeit vermittelt werden soll. Die Kotherapeuten haben dabei die Möglichkeit, neben Strategien der Gesprächsführung und Anleitung für Krisengespräche, verschiedene verhaltenstherapeutische Interventionen und auch die Durchführung verschiedener Gruppenangebote (z.B. Progressive Muskelrelaxation nach Jacobson) zu erlernen. Zudem nehmen die Kotherapeuten während der Ausbildung an Supervisionen teil. Neben den theoretischen Fortbildungen wird für das Zertifikat der Nachweis einer praktischen Tätigkeit über 2 Jahre gefordert.

\section{Gibt es wissenschaftliche Studien über die Effektivität von Kotherapeuten in der Verhaltenstherapie?}

Verschiedene Studien konnten positive Effekte in der Behandlung der Patienten nachweisen: Reasor und Farrell [2005] beispielsweise konnten in ihrer Einzelfallstudie eine deutliche Verbesserung sowohl der Zwangssymptomatik als auch der Depression eines Zwangspatienten nach einer erfolgten kognitiv-verhaltenstherapeutischen Behandlung mit Exposition, die durch eine psychiatrisch ausgebildete Krankenschwester durchgeführt wurde, belegen. Ekers und Kollegen [2011] fanden in ihrer randomisierten kontrollierten Studie signifikante Verbesserungen in der depressiven Symptomatik von Patienten nach einem Verhaltensaktivierungs- training, das von Personen aus dem Gesundheitssystem durchgeführt wurde, die zuvor nicht als Therapeuten tätig waren. Saur und Ford [1995] wiesen neben einer kostengünstigeren Behandlungsmethode ebenfalls die Qualität der Therapie durch geschulte Krankenschwestern nach.

Trotz der positiven Ergebnisse besteht jedoch kein Zweifel daran, dass verhaltenstherapeutische Interventionen nur dann erfolgreich ausgeführt werden können, wenn das Personal ein intensives Training erhalten hat [Zauszniewski et al., 2007].

\section{Werden die positiven Studienergebnisse bereits in der Realität umgesetzt?}

Die oben dargestellten Ergebnisse weisen darauf hin, dass Kotherapeuten aus verschiedenen Fachgebieten einen wichtigen Beitrag zur aktuellen psychiatrischen und psychosomatischen Versorgung von Patienten mit psychischen Störungen leisten können. Mit einer speziellen und qualifizierten Weiterbildung ist es ihnen möglich, verhaltenstherapeutische Interventionen wirksam durchzuführen und die Therapie zu unterstützen. Darüber hinaus ist die Behandlung durch geschulte Kotherapeuten häufig deutlich kostengünstiger, was ebenfalls für deren Einsatz spricht. Doch gerade die qualifizierten verhaltenstherapeutischen Aus- und Weiterbildungen, die theoretisches und praktisches Fachwissen vermitteln, stellen in der Realität häufig ein Problem dar; so gibt es derzeit keine einheitlichen Regelungen für den Erwerb einer Zusatzqualifikation, sodass eine flächendeckende professionelle Versorgung nicht zu gewährleisten ist. Daher ist es wichtig, den Einsatz von Kotherapeuten im Einzelfall zu bewerten und die individuellen Qualifikationen zu beachten.

\section{Literatur}

- Ekers D, Richard D, McMillan D, Bland JM, Gilbody $\mathrm{S}$ : Behavioural activation delivered by the non-specialist: phase II randomised controlled trial. Br J Psychiatry 2011;198:66-72.

Gourney K, Denford L, Parr A-M, Newell R: British nurses in behavioural psychotherapy: a 25 -year follow up. J Adv Nurs 2000;32:343-351.

Linden M, Gehrke G: Therapieziele und Therapieoptionen einer verhaltenstherapeutisch orientierten Ergotherapie. Verhaltenstherapie 2013 DOI: $10.1159 / 000348596$
Marks IM, Hallam RS, Phillpott R, Connolly, JC: Nurse therapists in behavioural psychotherapy. BMJ 1975;3:144-148.

Newell R, Gourney K: Britisch nurses in behavioural psychotherapy: a 20-year follow-up. J Adv Nurs 1994;20:53-60.

Reasor JE, Farrell SP: The effectiveness of advanced practice registered nurses as psychotherapists. Arch Psych Nurs 2005;19:81-92.

Saur C, Ford S: Quality, cost-effective psychiatric treatment: A CNS-MD collaborative practice model. Arch Psychiatr Nurs 1995;6:332-337.
Ward M: Psychiatric/Mental Health Nursing and Psychotherapy: The Position of Horatio: European Psychiatric Nurses, 2012. www.horatio-web.eu/ downloads/Psychotherapy_position_paper.pdf $(\mathrm{Zu}$ griff vom 11.02.2013).

Zauszniewski JA, Sursky MJ, Bekhet AK, Kidd LK Moving from tradition to evidence: a review of psychiatric nursing intervention studies. Online J Issues Nurs 2007;12:9. 